\title{
COMPARAÇÃO DO COMPORTAMENTO DOMINANTE DE FILHOTES DE CÃES DE CINCO RAÇAS
}

\author{
Guilherme Marques Soares ${ }^{1}$, João Telhado ${ }^{2}$, Rita Leal Paixão ${ }^{1}$ \\ 1 Universidade Federal Fluminense - gsoaresvet@gmail.com \\ 2 Universidade Federal Rural do Rio de Janeiro
}

RESUMO: Campbell, em 1972, desenvolveu um teste para seleção de filhotes de cães, com objetivo de determinar seu grau de dominância para direcioná-los para uma atividade ou família compatível. No presente estudo, o teste de Campbell, tal como ficou conhecido, foi aplicado em filhotes de cinco raças para avaliar possíveis diferenças comportamentais. Os filhotes (138 Labrador; 71 Rottweiler, 31 Bull Terrier, 13 Cocker Spaniel Inglês e 12 Pit Bull), com idade entre seis e oito semanas foram testados em seus canis de origem. Os resultados foram analisados estatisticamente através do teste de Kruskal-Wallis e a comparação entre os pares pelo teste de Mann-Whitney. Os filhotes de Labrador mostraram maior pontuação total no teste comparado aos de Rottweiler $(P=0,02)$ e Bull Terrier $(P=0,01)$. Com esses resultados pode-se caracterizar uma maior atração do filhote de Labrador ou uma maior independência do filhote de Rottweiler e de Bull Terrier em relação ao ser humano, considerando que as etapas do Teste de Campbell que mais evidenciaram a diferença foram àquelas em que o filhote tem que demonstrar interesse pelo examinador.

Palavras-chave: agressão, cães, dominância

\section{COMPARISON OF THE DOMINANCE BEHAVIOR BETWEEN FIVE BREEDS OF PUPPIES DOGS}

\begin{abstract}
In 1972, Campbell developed a test for selection of puppies, aiming to determine their degree of dominance to direct them to a compatible activity or family. In the present study, The Campbell test, as it became know, was applied to puppies from five breeds seeking possible behavioral differences. The puppies (138 Labrador retriever; 71 Rottweiler, 31 Bull Terrier, 13 English Cocker Spaniel and 12 Pit Bull), ranging from six to eight weeks of age, were tested in the kennels where they born. The results were analyzed statistically through the Kruskal-Wallis' test, and the comparison between pairs was done using the Mann-Whiteny's test. The Labrador Retrievers' puppies scored higher when compared to Rottweilers $(p=0,02)$ and Bull Terriers $(p=0,02)$. These results suggest a higher attraction of the Labrador puppy or a greater independence of the Rottweiler and Bull Terrier puppies in relation to human beings, whereas the steps of the Test of Campbell that showed the most difference were those in which the puppy has to show interest by the examiner.
\end{abstract}

Key Words: dogs, aggression, dominance 


\section{INTRODUÇÃO}

A necessidade de selecionar cães para diversas atividades ou famílias com aspectos distintos fez com que William Campbell desenvolvesse e publicasse um teste para seleção de filhotes (Campbell, 1972). Esse teste, que se tornou conhecido como Teste de Campbell, tem por objetivo avaliar o grau de dominância do filhote a fim de enquadrar o filhote testado no melhor no perfil de família que o está adquirindo ou facilitar a escolha da atividade mais adequada para esse filhote. A dominância é um traço individual de conduta, comum a indivíduos de várias espécies, inato e tem forte importância evolutiva para espécies sociais (Cameron, 1997). Essa característica faz com que o cão assuma postos mais altos em sua hierarquia social, sendo 0 conceito oposto à submissão (Beaver, 2001). Tal característica é manifestada nos cães de diversas maneiras, sempre com o objetivo de obter o controle dos recursos, que no ambiente doméstico são: alimento, abrigo, brinquedos, a "cama", atenção e carinho das pessoas. O cão dominante pode atuar de forma pacífica ou agressiva ao se aproximar de alguém, seja esse alguém um ser humano, um outro cão ou outro animal. $\mathrm{Na}$ abordagem agressiva, o cão comporta-se inicialmente com intimidações posturais ou sonoras e posteriormente com ataques direcionados àquele que ameace sua superioridade hierárquica ou a ordem natural da matilha (Overall, 1999). A agressão por dominância é o principal problema de comportamento em cães diagnosticado em serviços de etologia clínica por todo o mundo (Landsberg, 1990).

A questão racial dos cães é polêmica e gera diversos preconceitos sociais (Twining et al., 2000) e leis proibindo o trânsito ou a reprodução de algumas raças (Rio de Janeiro, 1999; Rio de Janeiro, 2005; Rosado et al., 2007). Mas a pergunta que este estudo se propõe a responder é se há realmente diferenças significativas na tendência à dominância entre as raças, a fim de munir os profissionais que trabalham com cães, como médicos veterinários, adestradores e criadores, de informações para prevenir problemas relacionados à agressividade por dominância.

\section{MATERIAL E MÉTODOS}

Foram selecionadas cinco raças, duas do grupo discriminado pela lei $\mathrm{n}^{\circ}$. 4597 do Estado do Rio de Janeiro (Rio de Janeiro, 2005) e duas raças tidas como cães de companhia, populares no Brasil, mas que aparecem em estudos internacionais como frequentemente envolvidas em ataques contra seres humanos, o Retriever do Labrador (Guy et al., 2001) e o Cocker Spaniel Inglês (Fatjó et al., 2007). A raça Bull Terrier foi inserida no estudo por sua semelhança fenotípica com o Pit Bull e por também ser citada pela literatura internacional como envolvida em ataques contra seres humanos (Overall e Love, 2001). A proporção do número de filhotes obedece à proporção de filhotes nascidos no período dos testes, e registrados pelo Kennel Clube Fluiminense (Labrador, 66; Rottweiler, 52; Cocker Spaniel Inglês, 16; Bull Terrier, 15, Pit Bull, 7). Ao todo, 265 filhotes foram submetidos ao Teste Comportamental para Seleção de Filhotes de Campbell (1972), 138 cães da raça Retriever do Labrador (77 machos e 61 fêmeas) e 71 cães da raça Rottweiler (35 fêmeas e 36 machos), 31 da raça Bull Terrier (15 fêmeas e 16 machos), 13 Cocker Spaniel Inglês (nove fêmeas e quatro machos) e 12 da raça American Pit Bull (sete fêmeas e 
cinco machos). Todos os cães tinham idade entre seis e oito semanas, foram oriundos de canis especializados em suas raças no estado do Rio de Janeiro e nasceram no período de março de 2008 a junho de 2009. Os testes foram realizados nos canis de origem e pela mesma pessoa em todos os filhotes. 0 examinador foi um médico veterinário com experiência na área de etologia clínica, que não tivera contato anterior com os filhotes até então e evitou exibir qualquer emoção durante os testes.

O teste de Campbell é dividido em cinco etapas, cada uma delas corresponde a uma manipulação feita com o filhote, na qual sua resposta é avaliada e classificada como muito dominante, dominante, submisso, muito submisso e independente/medroso. As etapas do teste são:

(A) Atração social: em uma área de no mínimo $3 \mathrm{~m}^{2}$, o examinador posiciona o filhote de frente para uma parede, se afasta cerca de um metro, se agacha e chama o filhote batendo palmas sem fazer muito barulho, observa se o filhote atende ou não esse chamado e como ele o faz. As respostas possíveis incluem: (dd) o filhote vem prontamente, de cauda erguida, buscando contato com o examinador; (d) o filhote vem prontamente, de cauda erguida e não faz contato com o examinador; (s) 0 filhote vem prontamente, de cauda abaixada; (ss) o filhote vem hesitante, de cauda abaixada; (i)o filhote ou não vem ou foge.

(B) Seguir o examinador: na mesma área da etapa anterior, o examinador posiciona 0 filhote encostado na parede, mas virado para $\mathrm{si}$, tendo certeza que tem a atenção do filhote e começa a andar para o centro da área, observando se 0 filhote 0 segue e como o faz. As possíveis respostas incluem: (dd) o filhote segue prontamente o examinador de pé, cauda erguida, tentando brincar; (d) o filhote segue prontamente o examinador de pé, cauda erguida; (s) o filhote segue prontamente o examinador com a cauda abaixada; (ss) o filhote segue hesitante; (i) o filhote não segue examinador ou foge dele.

(C) Contenção: o examinador posiciona o filhote em decúbito dorsal, o contém nesta posição por aproximadamente 30 segundos e observa sua reação. Respostas possíveis incluem: (dd) o filhote se debate vigorosamente mordendo ou rosnando, com a cauda balançando; (d) o filhote se debate vigorosamente, com a cauda balançando, sem morder ou rosnar; (s) o filhote se debate e depois se acalma; (ss) o filhote não se debate e pode lamber a mão do examinador.

(D) Dominância social: o examinador posiciona o filhote em decúbito ventral, em posição de esfinge, com uma mão o contém na nuca, com a outra afaga seu dorso por aproximadamente 30 segundos e observa a reação do filhote. Respostas possíveis: (dd) o filhote se agita, rosna ou tenta morder; (d) o filhote se agita, mas não exibe comportamento agressivo; (s) o filhote se agita por um curto período de tempo; (ss) o filhote assume o decúbito dorsal; (i) o filhote foge e não volta.

(E) Dominância Elevada: 0 examinador contém o filhote com as mãos em torno do tórax do animal e o levanta do solo aproximadamente $20 \mathrm{~cm}$ e observa a reação do filhote. Respostas possíveis: (dd) o filhote se debate vigorosamente rosnando ou tentando morder; (d) o filhote se debate, mas não exibe comportamento agressivo; (s) o filhote se debate depois se acalma e/ou lambe as mãos do examinador; (ss) o filhote não se debate e pode lamber as mãos do examinador.

Os escores nas diferentes etapas do teste indicam: (dd) dominância excessiva (5 pontos); (d) dominância (4 pontos); (s) submissão equilibrada (3 
pontos); (ss) submissão excessiva (2 pontos); (i) independência ou medo (1 ponto). A partir da análise dos resultados foram identificados os cães com maiores graus de dominância, estabelecendo-se um ranking entre os filhotes de forma decrescente. O teste de Campbell (1972), em sua versão original, não prevê pontuação para as manipulações que foram substituídos por números neste estudo para facilitar a aplicação dos testes estatísticos.

As pontuações foram tabuladas e todos os grupos foram comparados estatisticamente pelo teste de KruskalWallis e a comparação entre os pares pelo teste de Mann-Whitney através do programa BioEstat ${ }^{\circledR} 5.0$, com nível de significância de $5 \%(\alpha=0,05)$. O mesmo programa foi usado para realizar a estatística descritiva.

\section{RESULTADOS E DISCUSSÃO}

$\mathrm{Na}$ avaliação global (Tabela 1), os filhotes de Labrador obtiveram pontuação significativamente superior aos de Rottweiler $(P=0,02)$ e Bull Terrier $(P=0,02)$.

Os resultados do teste de KruskalWallis na pontuação para a etapa de "Atração Social" (Tabela 1) entre todas as raças apresentaram diferença significativa entre os resultados $(P=0,03)$. Na comparação entre os pares, pelo tesde de Mann-Whitney, os filhotes de Labrador apresentaram pontuação significativamente maior do que os de Rottweiler $(P=0,04)$. Os filhotes de Pit Bull apresentaram pontuação significativamente maior que os de Bull Terrier $(P=0,04)$. Nenhum dos demais pares apresentou diferença significativa.

$\mathrm{Na}$ segunda etapa do teste ("Seguir o Examinador"), os resultados apresentaram diferença significativa entre as respostas $(\mathrm{P}<0,01)$. $\mathrm{Na}$ comparação entre os pares (Tabela 1 ), os filhotes de Rottweiler apresentaram pontuação significativamente menor do que os todos os outros filhotes $(P<0,05)$.

$\mathrm{Na}$ comparação entre os filhotes de Labrador com os de Rottweiler, a maior pontuação dos primeiros aponta uma maior tendência à dominância, contudo ao se analisar os resultados etapa por etapa, vê-se que tal diferença numérica pode ser por causa de maior independência dos filhotes de Rottweiler, sugerida pela maior frequencia de cães com um ponto na etapa "Seguir o Examinador". Um ponto significa ao mesmo tempo independência ou medo do filhote, características diametralmente opostas. Por exemplo, pouquíssimos filhotes de Rottweiler demonstraram posturas características de medo durante os testes. Esses filhotes simplesmente não seguiam 0 examinador na segunda etapa do teste. A maioria o acompanhava com o olhar permanecendo atento, porém sem se deslocar ou então começava a cheirar o chão e se deslocava em outra direção, demonstrando independência. $\mathrm{Na}$ opinião dos autores, tal independência pode ser um indicativo de dominância.

$\mathrm{Na}$ etapa de "Contenção" (Tabela 1) houve diferença significativa entre as respostas $(P=0,04)$. Na comparação entre os pares, os filhotes de Cocker Spaniel Inglês tiveram pontuação significativamente maior que os de Labrador $(P<0,01)$. Os filhotes de Cocker Spaniel Inglês se debateram sem exibir reações agressivas com muito mais frequência que os filhotes de Labrador, que em sua maioria aceitaram a contenção em decúbito dorsal sem reações, o que justifica a maior pontuação dos primeiros. Nenhum dos demais pares teve diferença significativa.

$\mathrm{Na}$ quarta etapa do teste ("Dominância Social"), os resultados do teste de Kruskal-Wallis (Tabela 1) apresentaram diferença significativa entre os resultados $(P<0,01)$. $\mathrm{Na}$ com- 
Tabela 1 - Comparação das medianas dos resultados dos Testes de Campbell aplicados a filhotes de cinco raças

\begin{tabular}{|c|c|c|c|c|c|}
\hline 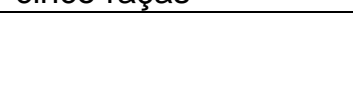 & $\begin{array}{l}\text { Retriever do } \\
\text { Labrador }\end{array}$ & Rottweiler & Bull Terrier & $\begin{array}{c}\text { Cocker Spaniel } \\
\text { Inglês }\end{array}$ & Pit Bull \\
\hline №. Filhotes & 138 & 71 & 31 & 13 & 12 \\
\hline Número de Ninhadas & 20 & 11 & 5 & 3 & 2 \\
\hline Etapas do teste & & & Medianas & & \\
\hline Atração Social & $4^{a}$ & $3^{\mathrm{a}}$ & $3^{b}$ & 2 & $4,5^{\mathrm{b}}$ \\
\hline Seguir o Examinador & $4^{e}$ & $1^{\text {cdet }}$ & $3^{d}$ & $4^{\dagger}$ & $3^{\mathrm{c}}$ \\
\hline Contenção & $2^{g}$ & 2 & 2 & $3^{g}$ & 2 \\
\hline Dominância Social & $2^{n}$ & $3^{\prime}$ & $1^{\mathrm{nj \textrm {j } k}}$ & $2^{1}$ & $3^{\mathrm{k}}$ \\
\hline Dominância Elevada & 2 & 2 & 2 & 2 & 2 \\
\hline Avaliação Global & $13^{\mathrm{Im}}$ & $12^{1}$ & $12^{\mathrm{m}}$ & 13 & 14,5 \\
\hline
\end{tabular}

Resultados das comparações entre pares a partir do teste de Mann-Whitney- a,b,c,d,f,l,m - P<0,05; e,k - P<0,001; g,j- $\mathrm{P}<0,01 ; \mathrm{h}, \mathrm{i}-\mathrm{P}<0,0001$

paração entre os pares, os filhotes de Bull Terrier tiveram pontuação significativamente menor do que os de Labrador $(P=<0,0001)$, de Rottweiler $(P<0,0001)$, de Cocker $(p<0,01)$ e de Pit Bull $(p<0,001)$. Nessa etapa ocorreu com os filhotes de Bull Terrier o mesmo fenômeno da segunda etapa entre os filhotes de Rottweiler e os demais. Os filhotes de Bull Terrier se comportaram de forma muito semelhante na quarta etapa do teste ("Dominância Social") e simplesmente saíam da posição de esfinge se deslocando para longe do examinador, mas sem exibição de sinais de medo. Essa independência segundo o autor do teste (CAMPBELL, 1972) deixa dúvidas quanto à dominância, mas a certeza da necessidade de um trabalho mais cuidadoso para a socialização desses filhotes. Esta contradição nos resultados poderia ser resolvida com uma reformulação do teste de Campbell, na relação de comportamentos esperados, separando respostas características de medo e independência, nas etapas de "Seguir o Examinador" e "Dominância Social", assim como na etapa "Atração Social".

$\mathrm{Na}$ quinta etapa ("Dominância elevada") poucos filhotes reagiram à manipulação, permanecendo praticamente inertes quando suspensos. Houve diferença significativa (Tabela 1) entre as respostas $(P=0,02)$ a partir do teste de Kruskal-Wallis, mas nenhum dos pares apresentou tal diferença de forma significativa.

O teste de Campbell é uma avaliação subjetiva e foi idealizado para ser feito pelo futuro proprietário do filhote para selecionar o animal que mais se adeque ao seu perfil. É um instrumento sujeito a variáveis como: examinador, ambiente hostil ou não e idade do filhote. No presente estudo tais variáveis foram minimizadas pelo fato de todos os testes terem sido realizados pela mesma pessoa e em ambiente não hostil aos filhotes, todos com idades entre seis e oitos semanas.

O teste tem como principal fator de restrição o fato de listar somente quatro ou cinco possibilidades de reação do filhote a cada manipulação proposta. Tal fato obriga a quem realiza o teste ter conhecimentos em etologia canina para enquadrar certos comportamentos apresentados ao mais próximo listado no teste. Por exemplo, na avaliação de "Dominância Social", houve filhotes que não se agitaram, não assumiram decúbito dorsal nem tentaram fugir, simplesmente permaneceram na mesma posição aceitando passivamente a manipulação.

Mesmo com as limitações, o teste não perde sua validade na comparação entre filhotes, por isso o teste tem sido aplicado em pesquisas científicas para avaliar dominância em filhotes (PérezGuisado et al, 2006). 
Nas etapas de "Contenção", "Dominância Social" e "Dominância Elevada", poucos cães exibiram comportamentos agressivos em qualquer dessas etapas, e tal reação foi independente de raça, ou seja, representantes de todas as raças reagiram agressivamente em algum desses testes, mas foram muito poucos. Caracterizando que essa resposta agressiva independe de raça em filhotes de seis a oito semanas.

Slabbert e Odendaal (1999) obtiveram bons resultados em um teste comportamental desenvolvido para selecionar animais para trabalho. Esses autores conseguiram prever em filhotes com oito semanas eficiência para treinamento de busca e com nove meses eficiência para prever agressividade. Apesar disso, os testes com filhotes de oito semanas parecem não ser eficientes em prever o comportamento dos animais quando adultos, de uma forma mais ampla, como no estudo realizado por Wilsson e Sundgren (1998). Isso ocorre, provavelmente, porque 0 resultado imediato do teste não prevê a reação deste filhote à forma com que será criado até a vida adulta nem a relação desse filhote com o proprietário, idéia já sugerida por Campbell (1972) ao divulgar o teste comportamental para seleção de filhotes. Corroborando esta idéia, um estudo espanhol (PérezGuisado e Muñoz-Serrano, 2009) identificou alguns fatores associados aos casos de agressão por dominância como: o fato do cão ter alimento a vontade; cães que caminham ou interagem pouco com seus proprietários; cães sem um processo de educação básica; e proprietários que tem um cão pela primeira vez. Todos esses detalhes devem ser considerados na seleção dos filhotes, associado ao teste, para assim prevenir problemas de agressividade por dominância nos cães, principalmente raças envolvidas no presente estudo por serem populares no Brasil.

\section{CONCLUSÃO}

A diferença, encontrada no presente estudo, entre os padrões de dominância dos filhotes de Retriever do Labrador, Rottweiler, Bull Terrierr, Cocker Spaniel Inglês e Pit Bull não é suficiente para afirmar se há uma raça com maior tendência à dominância do que outra, nem para estimar o comportamento desses cães quando adultos. Entretanto, sugere a necessidade de trabalhos de socialização diferenciados para as diferentes raças a fim de evitar manifestações agressivas desses cães.

\section{AGRADECIMENTOS}

Os autores agradecem a todos os criadores que permitiram a realização dos testes com seus filhotes.

\section{NOTA}

Esta pesquisa foi aprovada pelo Comitê de Ética para uso de animais em pesquisa da Universidade Federal Fluminense (protocolo ํㅡ. 0020/2008).

\section{REFERÊNCIAS}

BEAVER, B.V. Comportamento Canino: um guia para veterinários. São Paulo: Roca, 2001. $431 \mathrm{p}$.

CAMERON, D.B. Canine dominance-associated aggression: concepts, incidence, and treatment in a private behavior practice. Applied Animal Behavior Science, v.52, p.265-274, 1997.

CAMPBELL, W.E. A behavior test for puppy selection. Modern Veterinary Practice, v.53, n.12, p. 29-33, 1972.

FATJO, J.; AMAT, M.; MARIOTTI, V.M. et al. Analysis of 1040 cases of canine aggression in a referral practice in Spain. Journal of Veterinary Behavior: Clinical Applications and

Research, v.2, n.5, p. $158-165,2007$. 
GUY, N.C.; LUESCHER, U.A.; DOHOO, S.E. et al. Demographic and aggressive characteristics of dogs in a general veterinary caseload.

Applied Animal Behaviour Science, v.74, n.1, p.15-28, 2001.

LANDSBERG, G. Diagnosing dominance aggression. Canadian Veterinary Journal, v.31, p.45-46, 1990

OVERALL, K.L. Understanding and treating canine dominance aggression: An overview. Veterinary Medicine, v.94, p.975-978, 1999.

OVERALL, K.L.; LOVE, M. Dog bites to humans demography, epidemiology, injury, and risk, Journal of the American Veterinary Medical Association, v. 218, n. 12, p. 1923-1934, 2001. PÉREZ-GUISADO, J.; LOPEZ-RODRÍGUEZ, R.; MUÑOZ-SERRANO, A. Heritability of dominantaggressive behaviour in English Cocker

Spaniels. Applied Animal Behaviour Science, v.100, p. 219-227, 2006.

PÉREZ-GUISADO, J.; MUÑOZ-SERRANO, A. Factors Linked to Dominance Aggression in Dogs. Journal of Animal and Veterinary Advances, v.8, n.2, p.336-342, 2009.

RIO DE JANEIRO. Lei no 3.205, de 09 de abril de 1999. Dispõe sobre a importação, comercialização, criação e porte de cães da raça pit-bull, e dá outras providências.. Diário Oficial do Estado do Rio de Janeiro, Niterói, p. 1, 12 abr. 1999. pt.1. Disponível em:

<http://www.alerj.rj.gov.br> acesso 10 abril 2009
RIO DE JANEIRO. Lei no 4.597, de 16 de setembro de 2005 . Altera dispositivos da lei $n^{\circ}$ 3.205, de 09 de abril de 1999, e revoga a lei $n^{\circ}$ 3.207, de 12 de abril de 1999. Diário Oficial do Estado do Rio de Janeiro, Niterói, p. 4, 19 set. 2005. pt.1. Disponível em:

<http://www.alerj.rj.gov.br> acesso 10 abril 2009

ROSADO, B. et al. Spanish dangerous animals act: effect on the epidemiology of dog bites. Journal of Veterinary Behavior: Clinical Applications and Research, v.2, n.5, p.166174, 2007.

SLABBERT, J.M.; ODENDAAL, J.S.J. Early prediction of adult police dog efficiency-a longitudinal study. Applied Animal Behaviour Science, v.64, p.269-288, 1999.

TWINING, H.; ARLUKE, A.; PATRONEK, G. Managing the stigma of outlaw breeds: a case study of Pit Bull owners. Society and Animals, v.8, n.1, p.25-52, 2000.

WILSSON, E.; SUNDGREN, P.E. Behaviour test for eight-week old puppies-heritabilities of tested behaviour traits and its correspondence to later behaviour. Applied Animal Behaviour Science, v.58, p.151-162, 1998. 